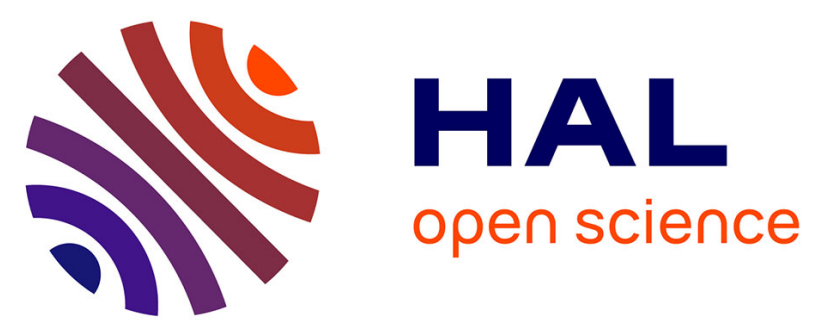

\title{
How to assess the benefits of connected vehicles? A simulation framework for the design of cooperative traffic management strategies
}

Maxime Gueriau, Romain Billot, Nour Eddin El Faouzi, Julien Monteil, Frédéric Armetta, Salima Hassas

\section{To cite this version:}

Maxime Gueriau, Romain Billot, Nour Eddin El Faouzi, Julien Monteil, Frédéric Armetta, et al.. How to assess the benefits of connected vehicles? A simulation framework for the design of cooperative traffic management strategies. Transportation research. Part C, Emerging technologies, 2016, 67, pp. 266-279. 10.1016/j.trc.2016.01.020 . hal-01298573

\section{HAL Id: hal-01298573 \\ https://hal.science/hal-01298573}

Submitted on 20 Mar 2019

HAL is a multi-disciplinary open access archive for the deposit and dissemination of scientific research documents, whether they are published or not. The documents may come from teaching and research institutions in France or abroad, or from public or private research centers.
L'archive ouverte pluridisciplinaire HAL, est destinée au dépôt et à la diffusion de documents scientifiques de niveau recherche, publiés ou non, émanant des établissements d'enseignement et de recherche français ou étrangers, des laboratoires publics ou privés. 


\title{
How to assess the benefits of connected vehicles? A simulation framework for the design of cooperative traffic management strategies
}

\author{
Maxime Guériau, Romain Billot, Nour-Eddin El Faouzi, Julien Monteil, Frédéric Armetta, Salima \\ Hassas
}

\begin{abstract}
Advances in Information and Communication Technologies (ICT) allow the transportation community to foresee dramatic improvements for the incoming years in terms of a more efficient, environmental friendly and safe traffic management. In that context, new ITS paradigms like Cooperative Systems (C-ITS) enable an efficient traffic state estimation and traffic control. C-ITS refers to three levels of cooperation between vehicles and infrastructure: (i) equipped vehicles with Advanced Driver Assistance Systems (ADAS) adjusting their motion to surrounding traffic conditions; (ii) information exchange with the infrastructure; (iii) vehicle-to-vehicle communication. Therefore, C-ITS makes it possible to go a step further in providing real time information and tailored control strategies to specific drivers. As a response to an expected increasing penetration rate of these systems, traffic managers and researchers have to come up with new methodologies that override the classic methods of traffic modeling and control. In this paper, we discuss some potentialities of C-ITS for traffic management with the methodological issues following the expansion of such systems. Cooperative traffic models are introduced into an open-source traffic simulator. The resulting simulation framework is robust and able to assess potential benefits of cooperative traffic control strategies in different traffic configurations.
\end{abstract}

Corresponding author: romain.billot@ifsttar.fr

Maxime Guériau and Nour-Eddin El Faouzi are with the Traffic Engineering Laboratory (LICIT), Université de Lyon, F-69000, Lyon, France, IFSTTAR, LICIT, F-69675, Bron, ENTPE, LICIT, F-69518, Vaulx En Velin.

Romain Billot is with Institut Mines-Télécom, Télécom Bretagne, UMR CNRS 6285 Lab-STICC, Technopôle Brest Iroise, CS 83818, 29238 Brest Cedex 3, France

Julien Monteil is with Trinity College Dublin, School of Computer Science and Statistics, Distributed Systems Group.

Frédéric Armetta and Salima Hassas are with the Laboratoire d'InfoRmatique en Image et Systèmes d'information, Université de Lyon, France. 


\section{How to assess the benefits of connected vehicles? A simulation framework for the design of cooperative traffic management strategies}

\section{INTRODUCTION}

\section{A. Context}

Cooperative Intelligent Transport Systems (C-ITS), a.k.a. connected vehicles, are new technologies that allow vehicles to communicate with other vehicles and with the infrastructure. The vehicle is equipped with bidirectional communication and sensors that enable to both capture and report on vehicles' surrounding traffic conditions and environment. More specifically, they refer to vehicle integrated systems that aim to provide the driver with a more comfortable and safer driving task. Vehicles are becoming more and more autonomous due to recent development of embedded technologies and Advanced Driver Assistance Systems (ADAS). Automated technology includes lane departure warning systems, emergency braking and adaptive cruise control (see [1] for a non-exhaustive review). In addition to autonomous technologies like ADAS, vehicles and infrastructure can be equipped with wireless communication devices. The recent developments of dedicated communication channels (e.g Dedicated Short Range Communication-DSRC, WIFI, WIMAX) increase the possibilities and amount of information potentially exchanged. Within these technologies, On-Board Units (OBU), Road Side Units (RSU), in-vehicle (ADAS) and on-road sensors (loop detectors, cameras) would play their key role [2]. The extensive use of the umbrella term "cooperative systems" in traffic-related research paper and projects is debatable as connected vehicles will obviously not cooperate with each other in the first deployment phases. Considering the advent of connected but not automated vehicles, cooperation can be seen as a combination of a high level of coordination and decentralization. The connected vehicle must be autonomous enough to ensure a self-decision process.

Such a communication framework looks very suitable for a decentralized approach, where vehicles are acting as mobile agents supplied by real time personalized and tailored recommendations, but also to a more centralized approach through the use of Road Side Units.

As new data sources as well as mobile sensors, connected vehicles revolutionize traffic management. Within connected vehicles environment, a twofold vision of vehicle is widely accepted:

1) as a probe delivering Connected Vehicle Data with extended capabilities;

2) as an actuator for traffic control.

Within the former, the vehicle will deliver rich and valuable information. Current probe data generated by vehicles deliver their current position, motion, and time stamp. Connected vehicles will enrich vehicle data with additional attributes such as headway, traction information, brake status, hard braking, activation of emergency lights, antilock brake status, air bag deployment status, windshield wiper status, etc. In addition, high resolution data such as vehicle trajectories may be provided. Probe data may be transmitted at various frequencies using a range of wireless communication technologies, including DSRC (Dedicated Short Range Communication), cellular, Wi-Fi, WiMAX, etc. In [3], we have proposed a queue-end detection algorithm on freeways, which is based only on the use of connected vehicle data. The results underline the capability of a simple algorithm to detect the end of a moving queue, i.e. a critical zone in terms of traffic safety, even with a low penetration rate $(15 \%$ of traffic). With respect to the second vision, the connected vehicle can be seen as an actuator for traffic control. Based on the information received in real time from all vehicles through Vehicle to Infrastructure communication (V2I), the Traffic Management Centre may send to the vehicle tailored and location-based recommandation or guidance through Infrastructure to Vehicle (I2V) communication. C-ITS enables the drivers more decisions, based on information from other vehicles and from the infrastructure. Different levels of interaction established by cooperative systems can potentially lead to changes in users behavior and ultimately to a Cooperative Traffic Management (CTM). However, the future deployment of these cooperative systems raises many issues and challenges in terms of modeling, network management and control which should be anticipated. Indeed, during the deployment phase of C-ITS, a situation of mixed traffic with connected and non-connected vehicles will perpetuate. Important developments are then necessary in order to achieve a Cooperative Traffic Management. In this respect, the European Network of Excellence NEARCTIS, led by our group, has contributed by identifying research gaps 
and elaborating an integrated research agenda and roadmap [4]. Unfortunatley, so far a comprehensive field data on connected vehicles is still limited and simulation studies are then crucial for understanding and modeling how to anticipate driver behaviour and the impacts on network flow dynamics, and how to use this information and multisource data to improve traffic control, which is a prerequisite for a better achievement of a Cooperative Traffic Management.

\section{B. Contributions and organization of the paper}

The objectives of this paper are to come up with new contributions anticipating and supporting deployment programs of connected vehicles. As an additional contribution to an extensive research in the past two years [5], [6], [7], [8], [9], the main point of our paper is the integration of our previous research efforts [10], [11], [12] into a robust decision support tool for cooperative traffic simulation. The paper summarizes the following research efforts:

- The integration of multi-agent cooperative traffic modeling into the MovSim Traffic simulator,

- The coupling of different dynamics (physical, informational) to account for sensors reliability issues,

- Simulation results about the impact of cooperative traffic on traffic flow homogenization and the effects of sensors failure,

- The design of RSU-based control strategies with on- and off- ramp scenarios.

The paper is organized as follows. Section II defines more precisely the concept of cooperative vehicle used in this paper and presents the multi-agent model (adapted from [10] ). In section III, the implementation of the model into MovSim is detailed. In section IV, simulation results are presented and then discussed in the final section.

\section{MULTI-AGENT COOPERATIVE MODELING (MACM)}

\section{A. Definition of a cooperative vehicle}

Cooperative Systems are an example of a networked world composed of mobile and fixed entities connected with each others. Information about traffic conditions and time-dependent vehicles parameters (speed, position) are exchanged through Vehicle to Vehicle (V2V), Infrastructure to Vehicle (I2V) / Vehicle to Infrastructure (V2I) communication, as described in figure 1.

Road Side Units (RSU) collect vehicle-based information to estimate and monitor traffic conditions along their covered road section. Exchanged information is not limited to kinematic parameters (speed, position, acceleration) but includes also the measurements stored by vehicles' sensors and trust values which represent the confidence vehicles have in others. In this work, the concept of cooperative vehicles can be understood as partially autonomous vehicles able to perceive their surrounding environment thanks to embedded sensors and act according to a cooperative self-behavior. In this approach, we consider that the driver is still in the loop and responsible for the steering behavior of its own vehicle but he delegates the longitudinal behavior to the cooperative automated system. As we focus on the situation of mixed traffic, figure 1.b presents the differences between cooperative $v s$. non-cooperative vehicles features.

Even though this is not the case here, drivers compliances could play a more important part in the system. Automation of the vehicle could also be extended to more complex behaviors as for intersection management. [15] propose an extension of Cooperative Adaptive Cruise Control (C-ACC) to include a cooperative behavior at intersections. The simulation framework allows to model such considerations but this issue is not addressed in the present paper. In our approach, cooperative vehicles longitudinal behavior is determined by applying an extended car-following law taking into account more than one vehicle ahead (and also several vehicles behind). In a previous work [11], we define this cooperative strategy as the Bilateral Multi-Anticipative model (BMA). The perception of non-connected vehicles is limited to their immediate leader (relative distance/speed) without any ability to communicate. The two physical models can be described as follows:

Non-connected vehicles: a generic car-following model gives the acceleration of the current vehicle $i$ as $\ddot{x}_{i}=f\left(\dot{x}_{i}, \Delta x_{i}, \Delta \dot{x}_{i}\right)$ where $f$ is a general non-linear function, $\dot{x}_{i}$ the current vehicle speed, $\Delta x_{i}$ the space headway (relative distance to immediate leader $i+1$ ) and $\Delta \dot{x}_{i}$ the relative velocity between current and leading 


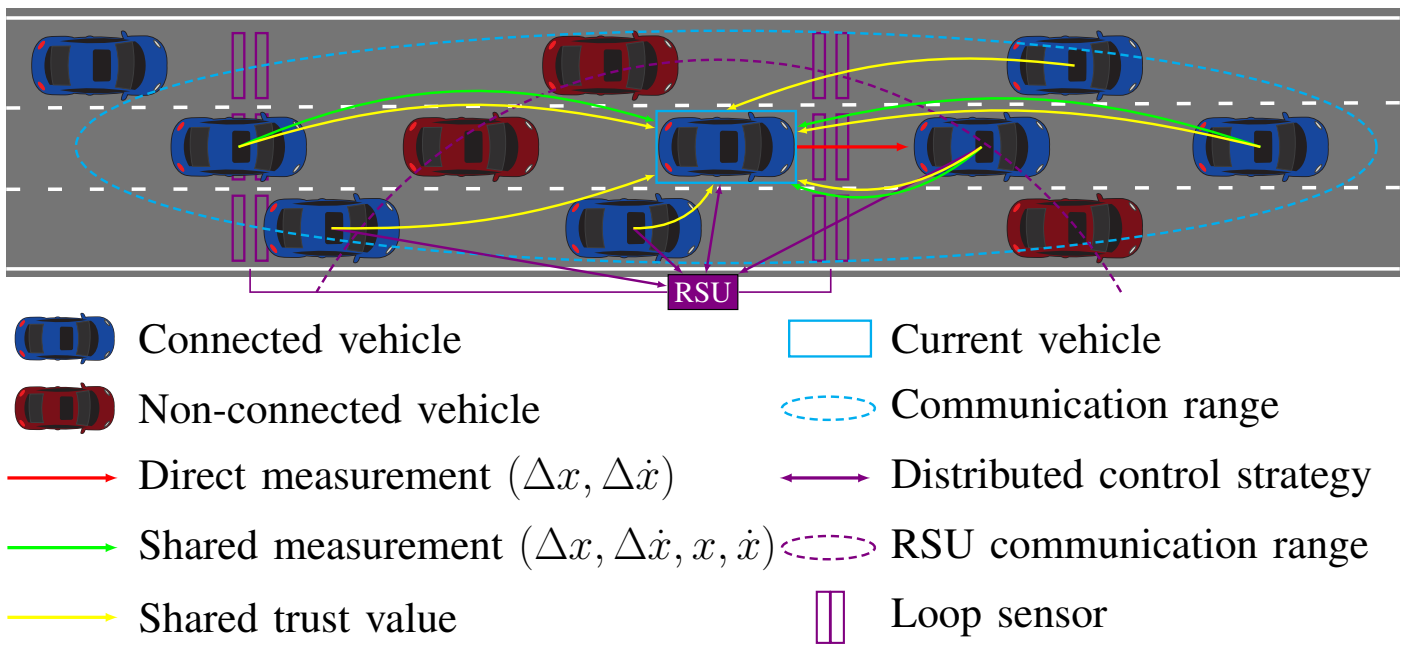

(a)

\begin{tabular}{|c|l|l|}
\cline { 2 - 3 } \multicolumn{1}{c|}{ Sensors } & \multicolumn{1}{c|}{ Non-cooperative vehicle } & \multicolumn{1}{c|}{ Cooperative vehicle } \\
\hline \multirow{2}{*}{ Exchanged information } & Nothing & $\begin{array}{l}\text { Long range laser telemeter } \\
\text { Wireless communication device } \\
\text { GNSS receiver }\end{array}$ \\
\hline Inherent information & $\begin{array}{l}\text { Instant speed } \\
\text { Speed restriction }\end{array}$ & $\begin{array}{l}\text { Sensor measurements } \\
\text { Trust values }\end{array}$ \\
\hline Sensed information & $\begin{array}{l}\text { Relative distance to leader } \\
\text { Relative speed with leader }\end{array}$ & $\begin{array}{l}\text { Instant speed } \\
\text { Speed restriction }\end{array}$ \\
\hline \multirow{2}{*}{ Physical model } & $\begin{array}{l}\text { Intelligent Driver Model [13] } \\
\text { Opportunistic lane-change strategy [14] }\end{array}$ & $\begin{array}{l}\text { Relative distance to n leaders } \\
\text { Relative speed with n leaders }\end{array}$ \\
\hline \multirow{2}{*}{ Control } & Opportunistic lane-change strategy [14] \\
\hline & Human Action & $\begin{array}{l}\text { Switchable (Cruise Control like) auto- } \\
\text { mated acceleration/braking control }\end{array}$ \\
\hline
\end{tabular}

(b)

Fig. 1: (a) General framework of Cooperative Systems. (b) Non-cooperative vehicle vs. cooperative vehicle comparison.

vehicles. We choose the Intelligent Driver Model (IDM), known as being collision-free and reproducing well instabilities in traffic flow [16]. This model is designed using braking and acceleration terms in addition to meaningful parameters. IDM function $f_{I D M}$ is written as

$$
\ddot{x}_{i}=a\left[1-\left(\frac{\dot{x}_{i}}{v_{0}}\right)^{\gamma}-\left(\frac{s^{*}\left(\dot{x}_{i}, \Delta \dot{x}_{i}\right)}{\Delta x_{i}}\right)^{2}\right],
$$

where $s$ is the current distance gap, $s^{*}\left(\dot{x}_{i}, \Delta \dot{x}_{i}\right)=s_{0}+\max \left(0, \dot{x}_{i} T+\frac{\dot{x}_{i} \Delta \dot{x}_{i}}{2 \sqrt{a b}}\right)$ is the desired distance gap, $a$ and $b$ are respectively the maximum acceleration and the desired deceleration, $v_{0}$ is the desired speed, $s_{0}$ is the minimum net stopped distance from the leader, $T$ is the desired safety time headway, and $\gamma$ is an acceleration exponent (usually set to 4). In addition, vehicles might perform an opportunistic lane change before updating their acceleration, according to the MOBIL strategy [17]. This strategy estimates if an available slot is safe enough by computing the time gap available on the nearest lane. The MOBIL strategy models the human lane-change behavior and includes a politeness parameter.

Connected vehicles: at a microscopic level, communication is modeled as a multi-anticipative car following model able to describe Vehicle to Vehicle (V2V) interactions and to improve traffic stability [18]. We use the bilateral multi-anticipation (BMA) model [11] as a combination of weighted influences from surrounding vehicles, 
written

$$
\ddot{x}_{i}=f_{I D M}\left(\dot{x}_{i}, \sum_{j} a_{i j} \Delta x_{i+j}, \sum_{j} a_{i j} \Delta \dot{x}_{i+j}\right)
$$

where $a_{i j}$ is the interaction coefficient of the weighted information between agent $i$ and perceived agent $j$ and $f_{I D M}$ is presented in (1). The interaction coefficient is computed from the proximity rule in the communication layer and the trust representation associated to each vehicle as:

$$
a_{i j}=p_{i j} \cdot T_{i j}
$$

where $p_{i j}$ represents the proximity rule (4), and $T_{i j}$ represents the trust $i$ has in $j$ (5). In [11], our research group has performed some stability analyzes of the BMA model. We have detailed a generic way of deriving stability domains and soliton equations for both non-cooperative and cooperative traffic. It is interesting to show how the fraction of cooperative (equipped) vehicles has an impact on traffic stability. This is a practical issue since the road operators are willing to know the minimal fraction (i.e. penetration rate) needed to stabilize traffic conditions.

\section{B. Multi-Agent Cooperative Traffic Modeling}

Modeling the complex microscopic behavior of cooperative vehicles implies to describe accurately the interactions of the systems at a local level, i.e. considering the point of view of one single vehicle. Artificial intelligence researchers brought such representation within complex decentralized systems called multi-agent systems. Agentbased simulation offers several benefits such as modularity, great flexibility and possible parallelization [19]. These benefits explain the interest for agent-based simulation and modeling applied to transportation [20]. The model to be implemented is adapted from [10]. Three layers couple different dynamics in order to take into account information reliability while limiting traffic disturbances, and hence homogenize traffic flow. Figure 2 presents the interaction between the three layers. The physical layer concerns the vehicle dynamics rules and estimates on its dynamics (microscopic car following models). The communication layer governs the information exchanges through proximity and reliability rules, which are expressed as probabilities. The trust layer models information reliability and enables an agent to evaluate its trust in others [21], [22]. Communication and trust layers influence the physical layer and hence vehicles behavior through the parameters of the bilateral multianticipative model.

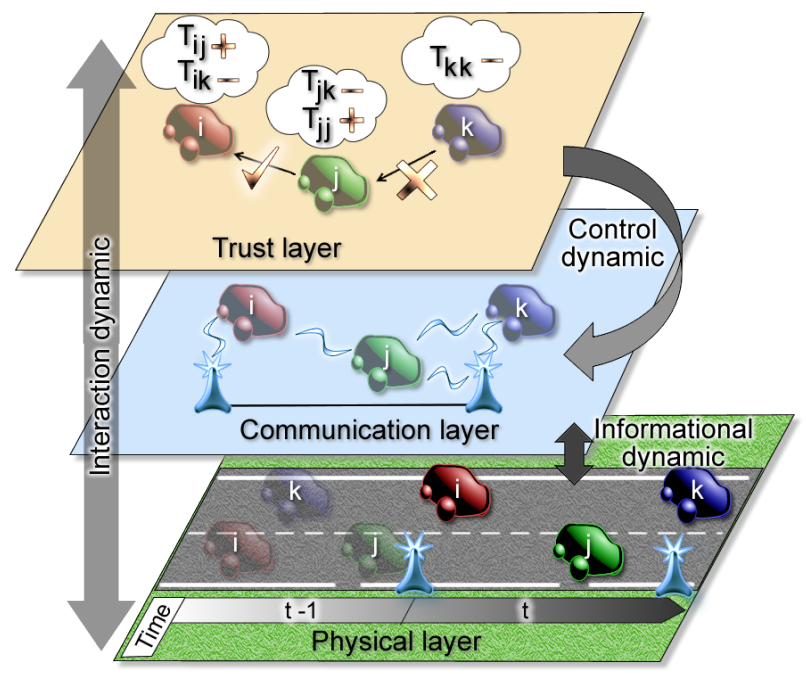

Fig. 2: Interacting layers

With respect to the communication layer, two kinds of message are shared by vehicles: measurement messages and trust messages. Hence the proximity rule $p_{i j}$ is written:

$$
P_{i j}=\frac{\left|\Delta \dot{x}_{i+j}\right|}{\left(\Delta x_{i+j}\right)^{\delta}}, \text { then } p_{i j}=\frac{P_{i j}}{\sum_{k \in \mathcal{P}_{i}} P_{i k}} .
$$


where $\mathcal{P}_{i}$ is the set of agents perceived by $i$ and $\delta$ controls forward and backward interaction (the chosen values are 2 and 0.5 respectively to ensure string stability and respect the physics of traffic flow). The idea is to give more importance to closer vehicles and/or vehicles with higher relative speed difference. Thus, connected vehicles exhibit anticipative behaviors and safety/homogeneity of vehicle string is maintained by the proximity rule. In the case of a communication failure where the current vehicle do not receive any information, the BMA model (2) will match the IDM non-cooperative car-following model (1).

Regarding the notion of trust, our work is influenced by the concept of delegation, which is critical in MultiAgent Systems [23]. Cooperative highway traffic is a perfect example of a delegation-based system since the action of a vehicle directly relies on the actions of other vehicles. A lot of trust and reputation models are available in the literature (see [24] for a review). We introduce here the trust concepts as a mechanism to cope with sensor reliability issues. We extend the trust model from [22], [25], which relies on the TrustNet data structure [21]. It provides a computational representation of trust based on non-aggregated experiences. In this model, truthfulness of agents rely on the majority, this is a known drawback which has no influence for traffic where average reliability is supposed to be trustworthy. Please note that selfish or cheating behaviors will not be considered here. Only the information reliability based on in-vehicle sensors failures will impact the trust an agent has in others (and in itself). During their decision process, agents compare their available information (embedded in trust messages). Then, each agent $i$ keeps and updates a local opinion of every previously perceived agent $j$ represented by a trust value $T_{i j}$

$$
T_{i j}=\frac{T_{i i} D T_{i j}+I T_{i j}}{T_{i i}+1} .
$$

This trust value is computed at every simulation time step $t$. It is formally a weighted average of three components $\in[0,1]:$

1) Direct trust $D T_{i j}$ : it represents the measurement error. It consists of assessing a percentage of error in terms of space headway as measured by vehicle $j$, written

$$
D T_{i j}=\frac{\left|\Delta x_{i}+x_{i}-x_{j}\right|}{\Delta x_{i}+x_{i}}
$$

where $\Delta x_{i}$ and $x_{i}$ correspond to the measurements of current vehicle $i$ (respectively from front telemeter and localization device), and $x_{j}$ is the position in global space shared by $j$ (given by its own localization device).

2) Indirect trust $I T_{i j}$ : it refers to the trust information sent by other agents in trust messages, or in other words, witness information. It is written

$$
I T_{i j}=\frac{\Sigma_{k \in \mathcal{W}_{i}} T_{i k} T_{k j}}{\Sigma_{k \in \mathcal{W}_{i}} T_{i k}},
$$

where $\mathcal{W}_{i}$ is the set of agents $k$ that share their trust value in $j$ with agent $i$ (for agents $k$ located in $i$ communication range). Thus, $k$ must have previously compared direct information with $j$, which helps at reducing significantly the complexity of the computation.

3) Self trust $T_{i i}$ : it is the average of the trusts that the other agents have in the considered agent $i$, at time $t$, hence taking into account the past information, written

$$
T_{i i}=\frac{\Sigma_{k \in \mathcal{C}_{i}} T_{k k} T_{k i}}{\Sigma_{k \in \mathcal{C}_{i}} T_{k k}},
$$

where $\mathcal{C}_{i}$ is the set of agents $k$ that share their trust in $i$ (for agents $k$ located in $i$ communication range). This self trust represents the confidence an agent has in itself.

As for the infrastructure part of cooperative systems, Road Side Units (RSU) are also considered as agents. Nevertheless, their behavior is not a physical behavior since their position is fixed along the road section. They rather ensure traffic monitoring and coordinated control on covered sections. This framework corresponds to a multi-agent system where mobile agents evolve in an environment shared with static agents in charge of control tasks. This approach has the advantage of being close to future applications of cooperative systems. 


\section{FROM A MICROSCOPIC TO A MULTI-AGENT TRAFFIC SIMULATOR}

\section{A. Integrating multi-agent cooperative modeling into traffic simulation}

The goal is to introduce the models proposed in [11], [10] into a generic and robust simulation platform. Microscopic traffic simulation has attracted a lot of research and developments of either commercial softwares or open-source platforms. Among the most-used simulators, one can cite SUMO [26], AIMSUN [27], VISSIM [28], TRAFFSIM [29] and MovSim [30]. Introducing cooperative vehicles shares some objectives with modeling vehicular ad hoc networks (VANETS) and a comprehensive review of simulators for VANETS purpose can be found in [31]. With respect to agent-based concepts, MATSim (Multi Agent Transport Simulation) is one of the only simulators exploiting agent concepts [32]. One can also mention ARCHISIM which relies on the definitions of agents behaviors to model drivers [33]. However, MATSim focuses more on traffic demand while ARCHISIM tackles the behavioural aspects. The main objective of our work is to simulate a cooperative traffic on a highway section, with possible on- and off-ramps. Hence the goal is more to compare traffic models than generate connected traffic at a network scale. As Open-Source platforms, SUMO and MovSIM are the most relevant candidates, highly portable and proposing a lots of features for comparing microscopic models. SUMO is widely used and the most cited all around the world whereas MovSim has been developed more recently. MovSim takes benefits from the most advanced concepts in traffic flow theory [34], [35]. Regarding V2V communication, one of the ongoing extensions of SUMO aims at simulating the effects of in-vehicle applications on drivers behaviours [26]. Moreover, the VEINS project is a recent extension of SUMO that offers a suite of models for IVC communication [36]. With respect to MovSim, it has been thought since the beginning to integrate such inter-vehicle communication [37], [38], [39]. Last but not least, the two platforms offer a clear choice in terms of programming language: C++ for SUMO vs. Java for MovSim. In our work, the Multi-model Opensource Vehicular-traffic SIMulator (MovSim) from [30] has been selected to be extended for cooperative traffic modeling, because of its generic design pattern and the several already implemented models. This simulator implements lane-based microscopic car-following models and has been developed as a decentralized system. Agent-based modeling is also of the interests of the authors [35]. This simulation model was implemented in Java and includes a graphical interface to visualize simulation results. The running simulator relies on the definition of XML scenarios that describe all simulation parameters (road network, traffic parameters, inflow, outputs). Although MovSim is a recently developed simulation platform, there are some attempts using MovSim as a simulation model for traffic flow. [40] uses simulated data from MovSim to improve traffic state prediction. [41] extend the simulator to study innovative platooning strategies relying on ITS technologies. Communication capabilities (Inter-Vehicle Communication - IVC) are used for jam anticipation in [37].

\section{B. Implementation framework}

MovSim original framework was a stable basis for our extension. We design a multi-agent framework on top of the existing architecture. As shown in figure $3 \mathrm{~b}$, we designed new objects and made the original classes inherit them in order to extend existing features.

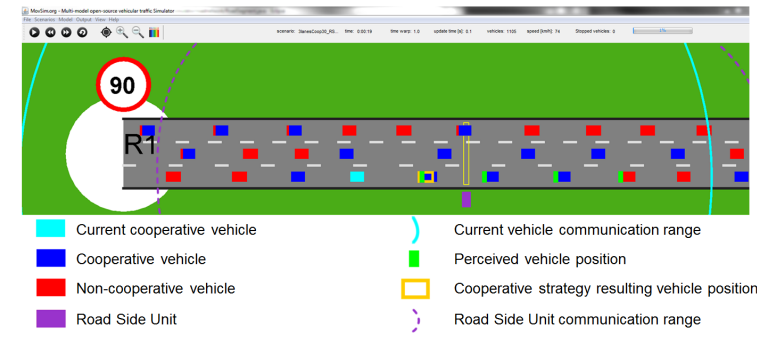

(a)

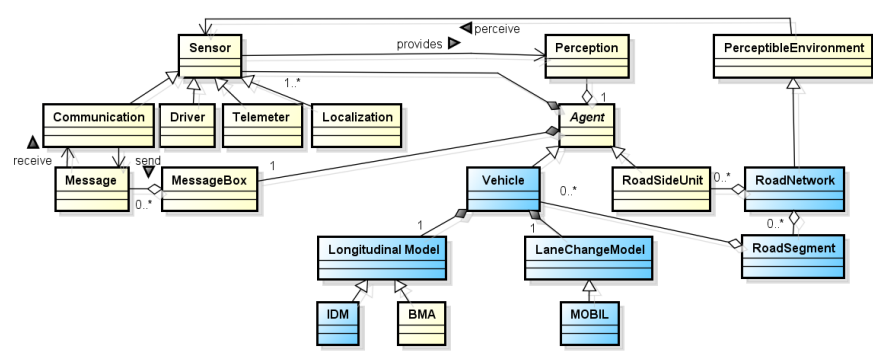

(b)

Fig. 3: (a) Screenshot of the cooperative traffic view in our MovSim. (b) Class diagram of the multi-agent framework integrated into MovSim. Blue classes are MovSim original ones. Yellow classes are our contributions.

Most of the changes are related to the implementation of perception and communication mechanisms. The original version of MovSim does include a graphical interface able to display a view of the running simulation with 
several micro (one vehicle parameters) and macro (inflow, outflow) indicators as well as the network (including vehicles) representation. Several coloration modes are available to focus on a specific aspect: occurring lanechanges, vehicle speed, kind of vehicle, accelerating/braking vehicles, etc. We have added some features dedicated to cooperative traffic simulation. This coloration makes the distinction between cooperative and non-cooperative vehicles, and gives a representation of the cooperative strategy with displayed communication ranges of both vehicles and RSU (as shown in figure 3a). Developers have also scheduled to create analytical graphs to visualize relevant indicators and highlight obtained simulation results. We extend the existing base by designing a spatiotemporal view representing vehicles trajectories (and speeds) over time. These diagrams are presented in the next section of this paper. They dynamically highlight the propagation of congestion waves and the effect of cooperative strategy on traffic flow homogenization. We refer the reader to [42] for a video presented at the AAAI 2015 demo track.

The environment was already at the center of the original framework and it is still the entry point of the simulation. With respect to the independence and autonomy of agents decision process (i.e. agents execution order should not affect the result of the simulation), the simulation relies on a scheduled time-discrete execution. Situated multi-agent systems rely on a perception-decision-action loop to ensure a temporal continuity between simulation steps [43]. The idea is to consider a fixed situation of the environment at a given time and then compute the corresponding perception for each vehicle. Then, each vehicle decides of an action to perform according to the environment sensed by embedded sensors and self-decision process. As a final step, the environment is updated by applying the vehicles' actions. This Sense-Decide-Act triptych will be developed in the following (figure 4).

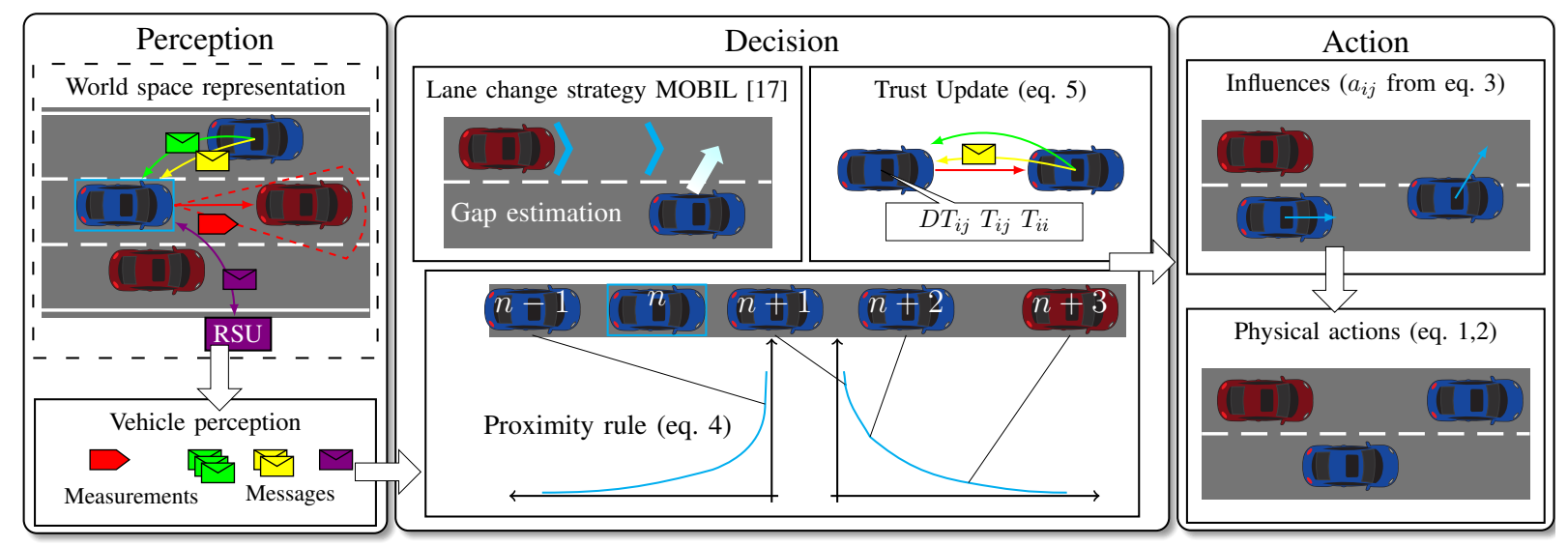

Fig. 4: Flowchart diagram of the simulation.

Perception: Thanks to embedded sensors (front long range laser telemeter, GPS receiver) and communication devices, cooperative vehicles can build an extended perception of the environment.As in multi-agent systems, cooperative vehicles have a partial and local representation of their surrounding environment. When new information is provided by direct measurements (space headway and relative speed), the vehicle performs a fusion of the information coming from both sensors and communication messages (cooperative vehicles share their own measurements). The resulting local representation is different for each vehicle.

Decision: Each cooperative vehicle (or reactive agent) has its own decision process. After a perception step, each vehicle handles recently received messages and extends this perception. Messages can also contain safety information or instructions from infrastructure (RSU). Road Side Units could be seen as static agents spaced along the controlled section and in charge of gathering information on their dedicated area in order to dynamically broadcast advices to cooperative vehicles and drivers. It can consist in adapting speeds and headways to current road conditions and learned recurrent perturbations. The decision maker is a partially automated vehicle, the drivers compliance is not taken into account.

Action: Non connected vehicles apply the IDM model (1) whereas connected vehicles follow the BMA model (2). Original MovSim version includes a model for lanes changes, MOBIL (Minimizing Overall Braking 
decelerations Induced by Lane changes [14]), which allows the vehicle to perform an opportunistic lane change according to target lane occupancy. This model is used to model lane-changes for both cooperative and non cooperative vehicles.

\section{Simulation Results}

The operational goal of this work is to come up with a decision-making tool validating under simulation conditions tailored cooperative strategies. All model parameters are randomly picked from a calibrated set [44] to reproduce drivers variability. We choose to focus on a suburban highway where it is likely that small perturbations propagate upstream (NGSIM data). It consists of a high density flow (40 veh/lane/km) evolving on a $10 \mathrm{~km}$ stretch with three lanes. The traffic is randomly initialized to fit with the chosen density. Vehicles start with a speed set to $90 \mathrm{~km} / \mathrm{h}$ (corresponding to the speed restriction of the section). Input flow is kept at $2000 \mathrm{veh} / \mathrm{h}$ during the 30 min simulation. Cooperative vehicles sensors are defined as reliable i.e. their sensors measurement have a maximal standard deviation of $5 \%$. The objectives of the proposed simulations are two-fold:

1) Assessing the impacts of the percentage of cooperative vehicles and the information reliability in a dense traffic,

2) Designing traffic management strategies based on the system's actors (RSU and connected vehicles).

\section{A. Introducing cooperation to enhance traffic flow dynamics}

Traffic flow homogenization: Figure 5 shows the vehicles trajectories for the defined scenario and different fractions of cooperative $v s$. non-cooperative vehicles. The diagram $5 \mathrm{a}$ is the reference simulation with $0 \%$ of cooperative vehicles.

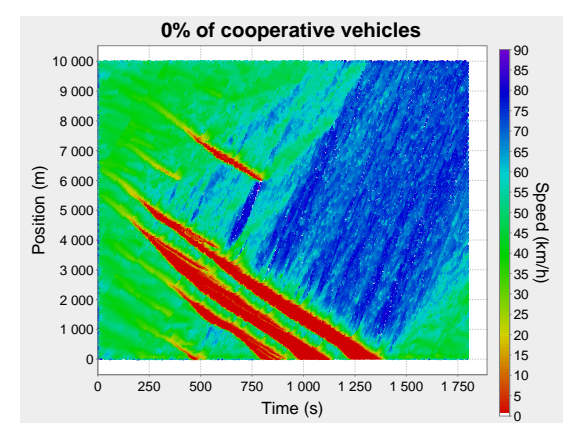

(a)

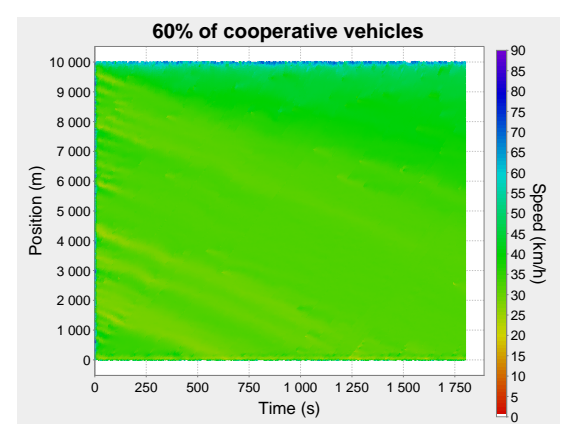

(d)

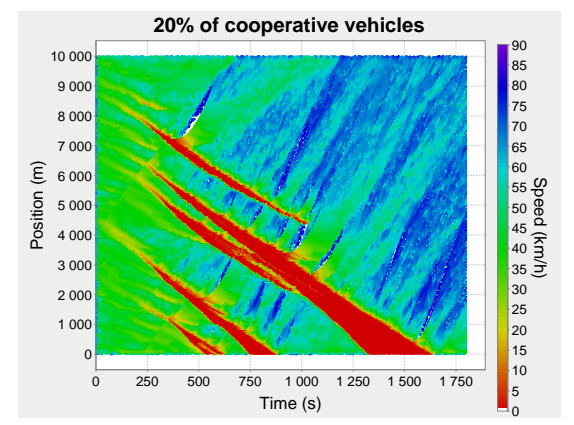

(b)

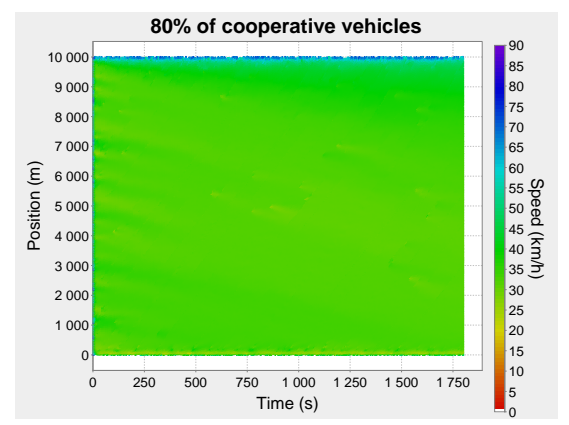

(e)

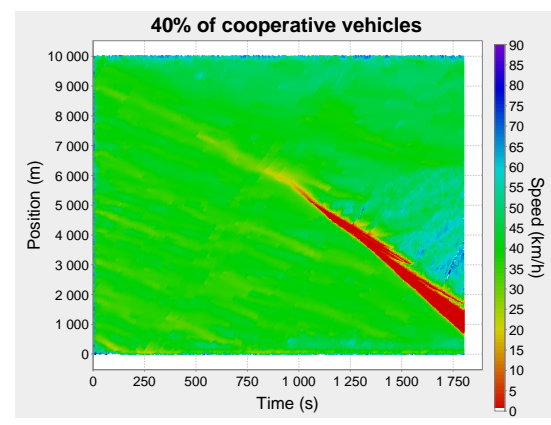

(c)

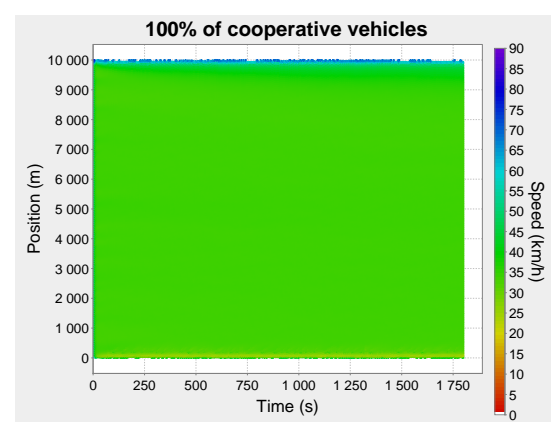

(f)

Fig. 5: Vehicles trajectories for several penetration rates. (a) $0 \%$ (b) $20 \%$ (c) $40 \%$ (d) $60 \%$ (e) $80 \%$ (f) $100 \%$

We observe that small perturbations create stop and go waves which propagate. Such instabilities result into a more heterogeneous traffic. While increasing the penetration rate (from $20 \%$ - fig. 5.b - to 100\% - fig. 5.f) of cooperative vehicles, i.e. the fraction of equipped vehicles, a cooperative traffic appears more resilient to the propagation of such instabilities. With higher fractions of cooperative vehicles (more than $40 \%$ in this scenario), the anticipation leads to a homogeneous traffic where perturbations are smoothed by cooperative behaviors. Note 
that with a high amount of cooperative vehicles, only less lane changes occur because the vehicles are organized around an equilibrium state (homogeneous speeds and headways).

Comparison with Adaptive Cruise Control: Adaptive Cruise Control systems (ACC) automates the longitudinal driving task by reacting to sensor inputs (relative distance and speed to immediate leader). We choose to compare our approach with this system to highlight the benefits of taking more than one vehicle into consideration within the car-following behavior. MovSim contains an ACC model designed in [34]. In this experiment, we make the comparison between a flow partially composed of cooperative vehicles $v s$. a flow of vehicles equipped with ACC. Initialization parameters (inflow, density, speeds) are the same as the previous case.

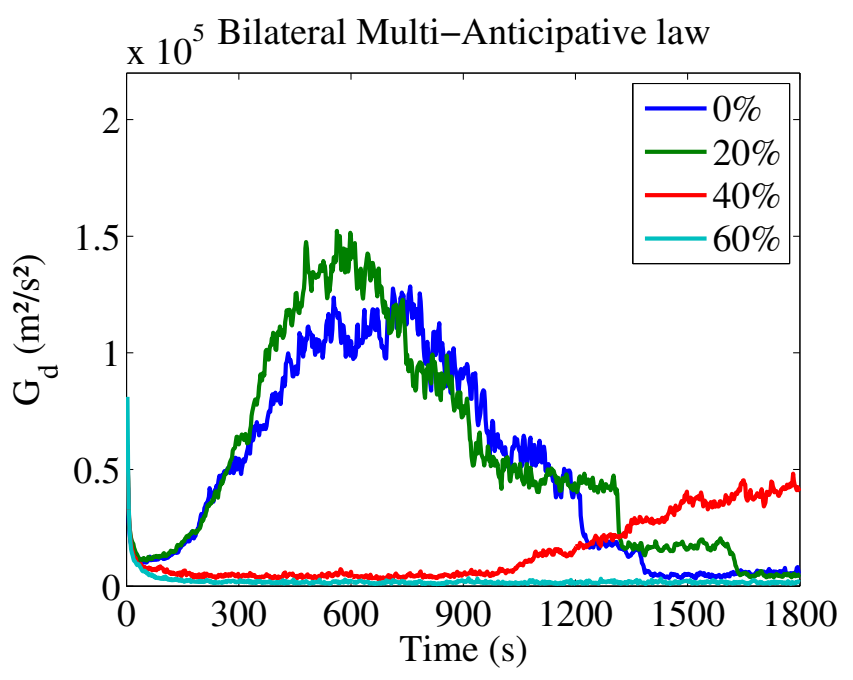

(a)

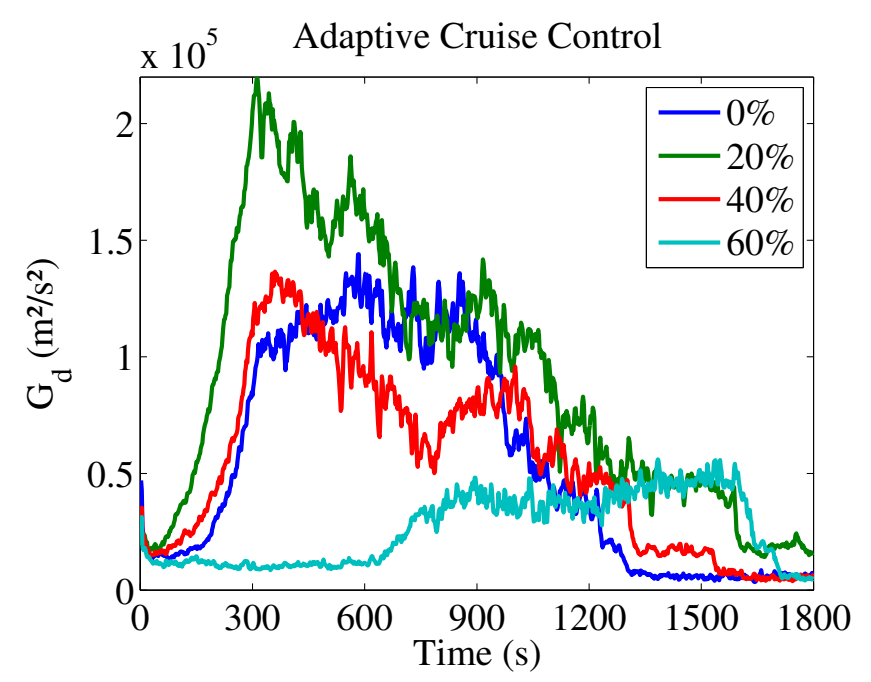

(b)

Fig. 6: Group disagreement values for several penetration rates: bilateral Multi-Anticipative law vs. ACC.

Figure 6 shows the Group Disagreement index for several fractions of equipped vehicles (Bilateral MultiAnticipative law - left - and Adaptive Cruise Control - right). A group disagreement value $G_{d}$ [45] is written

$$
G_{d}=\sum_{(i, j) \in \mathcal{N}^{2}} b_{i j}\left(\dot{x}_{j}-\dot{x}_{i}\right)^{2}
$$

where $b_{i j}$ is the binary adjacency value ( 0 if not in the communication range and 1 otherwise), and $\mathcal{N}$ is the set of vehicles on the road section. The $G_{d}$ index is relevant for evaluating homogeneity on a section with a comparable number of vehicles. A $G_{d}$ value of 0 represents a perfect homogeneous flow. In terms of homogenization, the defined cooperative strategy presents a higher impact than ACC for low penetration rates. For instance, we can see that the multi-anticipation effect delays the congestion apparition with $40 \%$ of cooperative vehicles (this is also visible on trajectories previously shown in figure 5.c). Moreover, transition between congested and free flow states appears smoother with the cooperative strategy than the ACC system. These results must be confirmed by different initialization contexts. In the future, the approach should also be compared with the cooperative extension of Adaptive Cruise Control (C-ACC), as tested in [46], as well as other relevant efforts about this topic such as [47].

Trust and sensors reliability: here the goal is to highlight the trust effect and to show how trust captures sensors drifts. Hence, some connected vehicles are equipped with perturbed sensors that provide high error values (+/- 50\%) for headways and relative speeds. Figure 7 depicts the distributions of the PET values for three different models : a model without trust, FIRE model and TrustNet model. We choose to compare our approach with the FIRE model [48], because both are computational trust models, suited to agent-based computation, with nonaggregation mechanisms to convert interactions between agents into trust values. Agents using FIRE store the results $r_{i}$ of past experiences in a finite memory. Each result is an evaluation of an interaction between two agents at a given time, similarly to our direct trust concept and the error measurement. We choose a memory limited to 
6000 interaction results (which corresponds to $10 \mathrm{~min}$ of simulation time with a $0.1 \mathrm{~s}$ time step). The trust value is computed from the weighted sum of past experiences, and the weights are decreasing along simulation time. The idea is to give more importance to recent interactions and to keep a memory of past interactions (the reputation module of FIRE is not implemented here). The trust value $T_{i j}$ is then evaluated as the weighted average of past evaluated interactions:

$$
T_{i j}=\sum_{r_{i}} \omega\left(r_{i}\right) \cdot \nu_{i}
$$

where $\omega\left(r_{i}\right)=\exp -\frac{\Delta t\left(r_{i}\right)}{\lambda}$ is the rating weight function that calculates the relevance or the reliability of the rating and $\nu_{i}$ is the value of the rating $r_{i}$. As described in the FIRE model, we choose $\lambda=-\frac{60}{\ln (0.5)}$ which implies that $\omega\left(r_{i}\right)=1$ after 0 second and $\omega\left(r_{i}\right)=0$ after 10 minutes (corresponding to agent maximum memory). We refer the reader to [48] for more details about the parameters tuning. In figure 7, we remind that the percentage
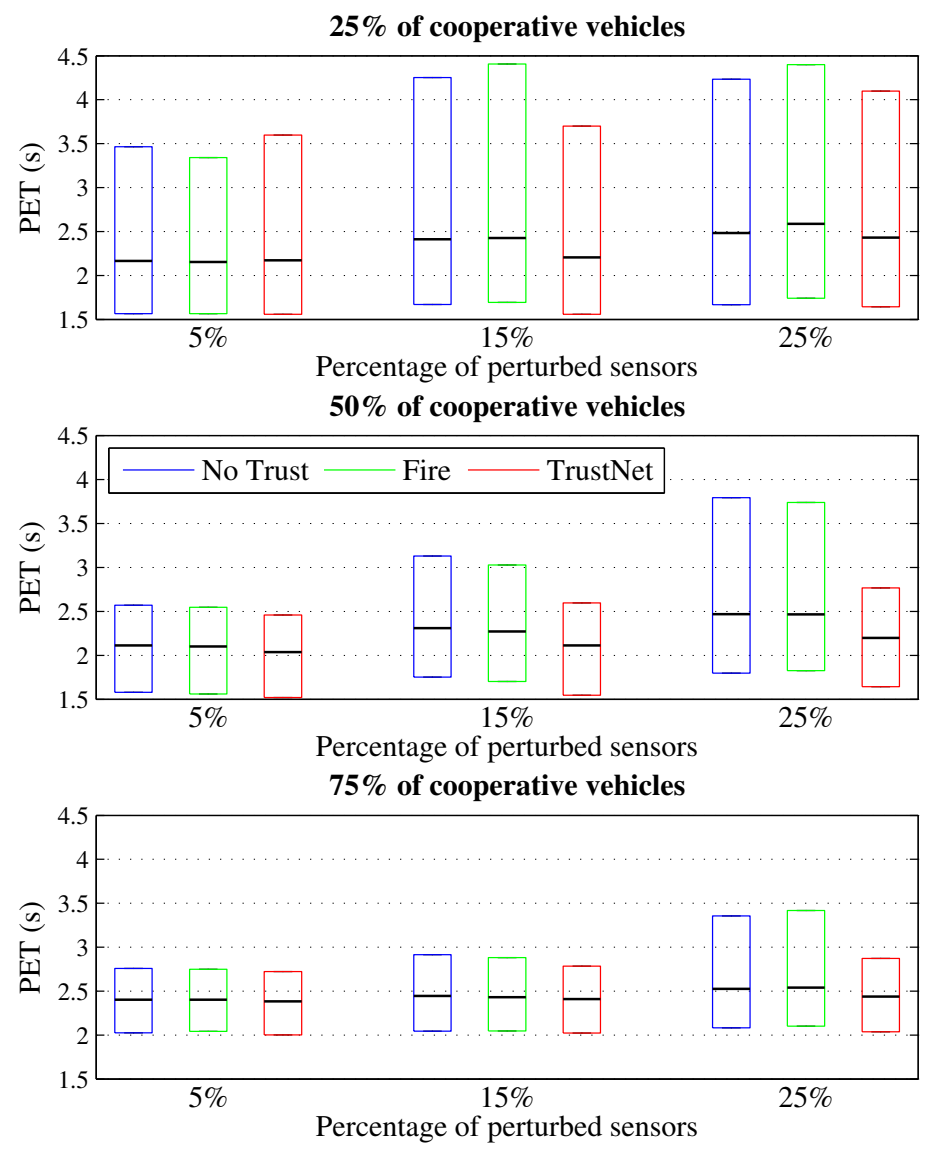

Fig. 7: PET distributions (median, $1^{\text {st }}$ and $9^{\text {th }}$ decile) for three trust variants. Top-down: increase of the $\%$ of equipped vehicles. Left-right: increase of the $\%$ of perturbed sensors.

of perturbed sensors only concern the connected vehicles. Hence, if this value is set to $25 \%$ with a fraction of equipped vehicles set to $25 \%$, it means that all the connected vehicles have perturbed sensors. With cooperative traffic, the goal would be to have homogeneous and safe PET values. In the top-down direction, figure 7 depicts an increase of our models performances when the percentage of connected vehicles increase. In general, the multianticipative car-following model enables an homogenization of the PET values, whatever the trust concept is, as well as a slight increase of the PET values. The results show that the trust layer reinforces this homogenization effect. Our TrustNet approach provides the best performances. In the left-right direction, it can be seen that the 
higher the fraction of perturbed sensors is, the better our approach outperforms the no-trust and FIRE approaches, even though the FIRE approach is also attractive given its simplicity.

\section{B. Design of control strategies}

This second part aims at using Road Side Units for designing tailored control strategies. Road Side Unites are in charge of collecting information in order to estimate the current traffic state on their dedicated section and hence disseminate warning message to reachable connected vehicles. The message broadcasted by the RSU includes information related to the location (lane, longitudinal position and radius) and the type of event (lane closure, on-ramp). When the information remains relevant (i.e. the current vehicle is located within the event radius), the message is retransmitted upstream. This dissemination ensures that connected vehicles get the information soon enough to launch a responsive action. We choose to provide connected vehicles with a lane changing advice in order to avoid a potential traffic jam occuring downstream. In this simulation, we assume that all vehicles which get the warning message are trying to change lane until they reach a safer one (100\% drivers compliance). Two scenarios are presented: on-ramp and lane closure.

On-ramp scenario: in this case, a merging section is represented and the experimentation consists in simulating $1000 \mathrm{veh} / \mathrm{h}$ for all lanes during 20 minutes. Figure 8 represents a screenshot of the traffic state after 17:03 minutes.

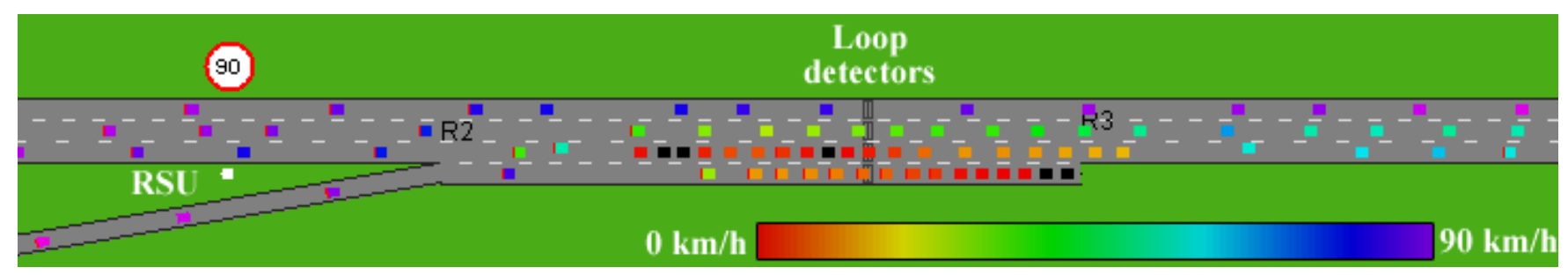

(a)

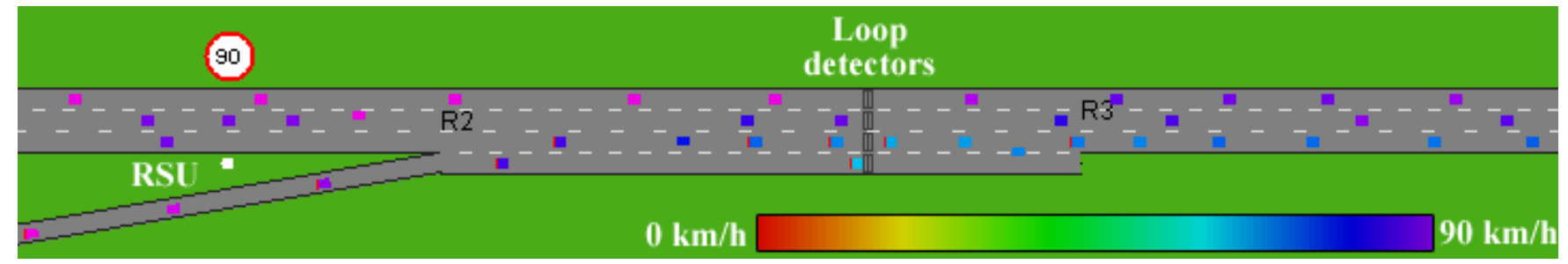

(b)

Fig. 8: On-ramp scenario. (a) traffic instabilities with $0 \%$ of connected vehicles (b) $40 \%$ of connected vehicles.

In figure 8a, we can see a typical merging case where incoming vehicles can not reach the main section, resulting into a moving jam. The on-ramp, the lane 3 and lane 2 (middle lane) are progressively getting congested. Connected vehicles may have the potential to improve these kinds of critical traffic conditions. In figure 8b, the white square represents the position of a RSU located upstream the on-ramp. The RSU sends a warning message to connected vehicles. The message gives the location of the critical point (here the on-ramp), a broadcast advice (here vehicles are broadcasting the information during the entire simulation) and a driving advice. Here, the order is to apply a mandatory lane change (when possible) instead of an opportunistic lane change as proposed by the MOBIL model [14]. Hence connected driving behaviors will consist of switching toward lanes 1 and 2 and to stay there until the perturbation is over. From $8 b$, it is clear that only $40 \%$ of connected vehicles within the entire flow are enough to keep a free-flow regime at the same simulation time. As depicted in figure 8, loop detectors have been added to the experimentation in order to validate the graphical observation. Figure 9 draws the different fundamental diagrams for all lanes.

This figure confirms the benefits coming from the RSU control strategy. The congested part of the fundamental diagram is never reached when the targeted advice is sent to the $40 \%$ of connected vehicles.

Lane closure scenario: the second scenario deals with a configuration of great interest for traffic operators. The section is restricted from 3 to 2 lanes and vehicles on lane 1 will try to move onto lane 2 as soon as they can. 

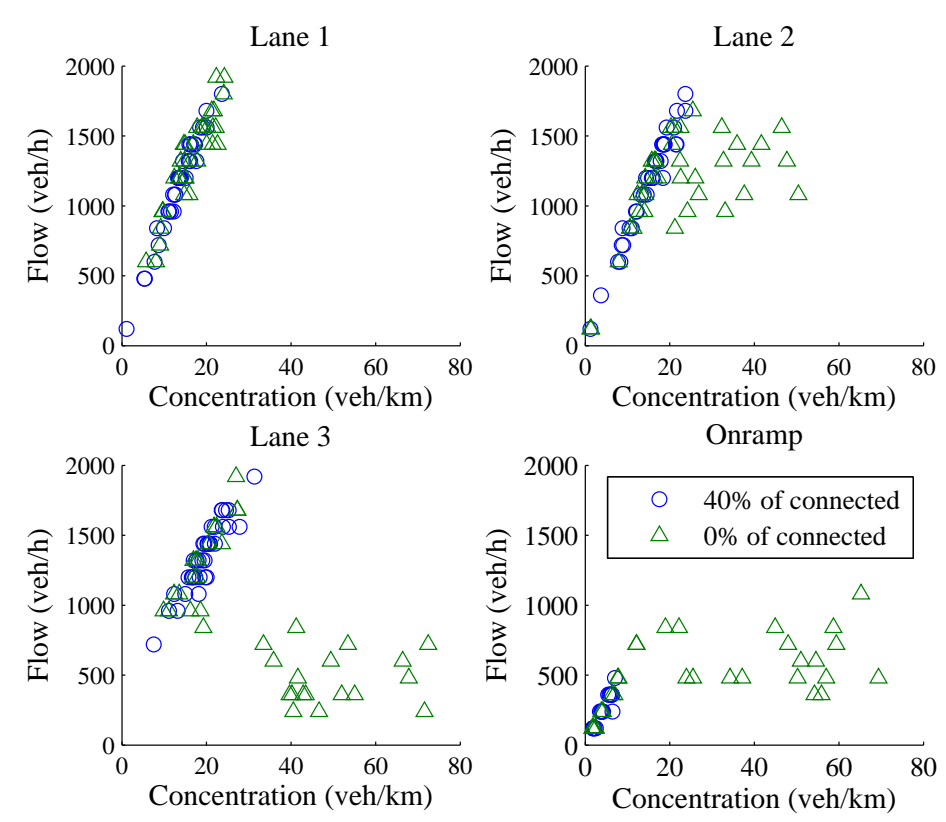

Fig. 9: On-ramp scenario. 1min Loop fundamental diagrams. (a) lane 1 (b) lane 2 (c) lane 3 (d) on-ramp

It will typically impact the two other lanes as shown in figure 10 where a snapshot is taken at time $t=15: 13$ minutes. The control strategy applied by the Road Side Unit remains the same.

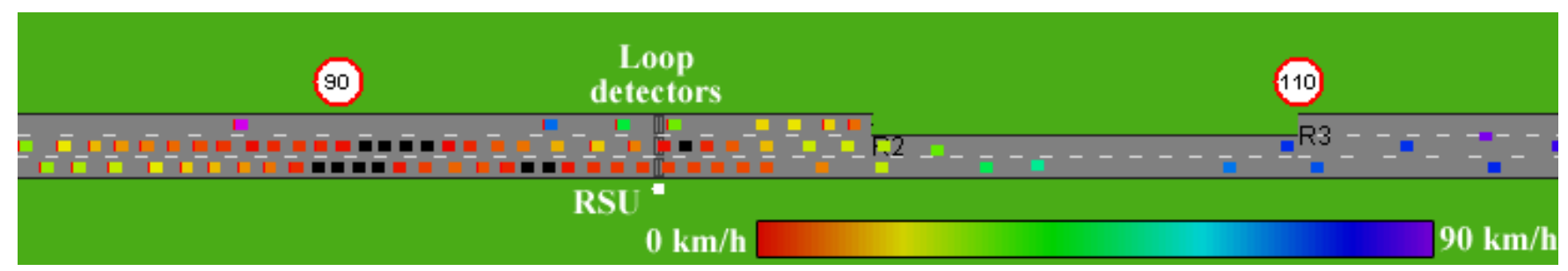

(a)

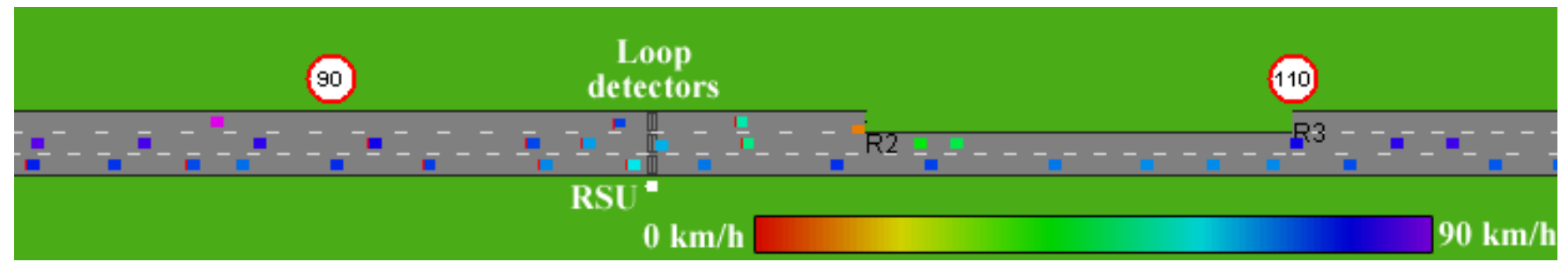

(b)

Fig. 10: Lane closure scenario. (a) traffic instabilities with $0 \%$ of connected vehicles (b) $50 \%$ of connected vehicles.

Vehicles on lane 2 will be forced to move onto lane 3 in order to leave more space for lane 1 vehicles. In the proposed experimentation, this simple advice (followed by $100 \%$ of the $50 \%$ connected vehicles) will solve the problem. In figure 11, fundamental diagram confirm the improvement on lane 2 and 3 while lane 1 was not impacted in both cases.

Discussion: All issues are not solved with such a simple control strategy which can be seen as a proof of concept. The presented RSU control strategy is based on strong assumptions that need to be addressed in a future work:

- the results are based on a given input flow and a systematic control algorithm should be developed in order 

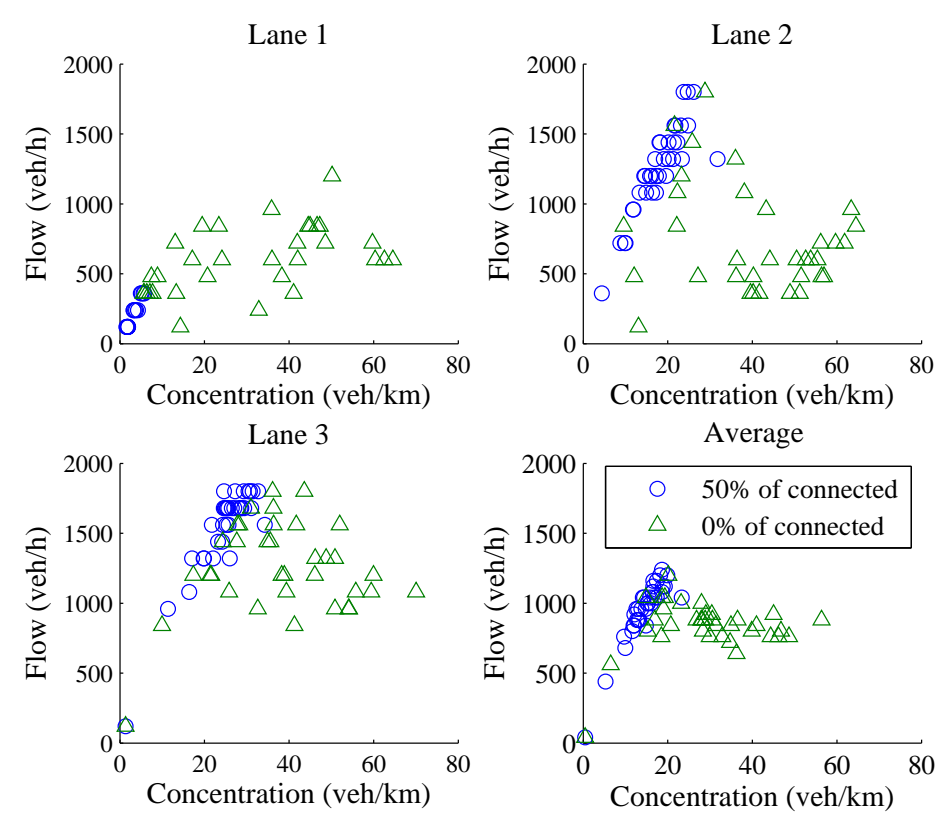

Fig. 11: Lane Closure scenario. 1min Loop fundamental diagrams. (a) lane 1 (b) lane 2 (c) lane 3 (d) average

to generalize them to all traffic situations. Such an algorithm must cope with various traffic densities and differents kinds of traffic heterogeneity (connected and non connected vehicles but also trucks, weather conditions, etc.).

- The RSU only repeats a single message to all connected vehicles with fixed parameters, without any dynamic adaptation.

- A $100 \%$ driver compliance has been assumed. Sensitivity analyzes are required to consider more realistic cases.

Nevertheless, these first results are promising for the use of RSU are semi-centralized sections controllers. In terms of implementation into the MovSim simulator, the message architecture is very flexible and one can imagine various messages types for various traffic conditions.

\section{CONCLUding REMARKS}

We have proposed a multi-agent based extension of MovSim to model complex interactions between cooperative vehicles and, potentially, to and from the infrastructure. The implemented framework models both communications (information dynamics) and physical interactions. The additional control using the concept of trust improves the robustness of the model against perturbations introduced by sensors unreliability. This aspect is critical in such applications where the acceptance of the system is the preamble to a massive deployment. First simulation results make us expecting benefits from the cooperative strategy, in terms of homogenization and safety in sections equipped with such technologies. The simulation model presented in this paper prefigures a decision support tool for the deployment of cooperative systems.

So far, the cooperative strategy reduces vehicles speeds in addition to an homogenization of both speeds and headways. This strategy could be enhanced by considering different cases according to traffic conditions (congested states, free flow situations) in order to adapt the behavior to observed conditions. Hence, we can expect that speed will be increased with the growing proportion of cooperative vehicles, without any bad impact on traffic safety (harmonized headways and smoothed braking to avoid perturbation appearance).

As for the design of traffic management strategy, this work has highlighted the strong potential of road side units. As mesoscopic agents, RSU enable a decentralized control strategy. It relies on the collection of information from vehicles to RSU which will be able to make an estimation of the current road conditions. Further to these first results, the next step if to develop a learning strategy. We expect that RSU will be able to send recommendations 
to vehicles (and/or drivers) in accordance to the determined context and the memory of past situations, responses and results.

\section{ACKNOWLEDGMENT}

Part of this work was supported by a grant from Rhone-Alpes Region Council through the Academic Research Program "Innovations, Mobilities and Territories Dynamics".

\section{REFERENCES}

[1] M. Lu, K. Wevers, and R. Van Der Heijden, "Technical feasibility of advanced driver assistance systems (adas) for road traffic safety," Transportation Planning and Technology, vol. 28, no. 3, pp. 167-187, 2005.

[2] C. . C. communication consortium, 2008.

[3] T.-U. J. Dinh, R. Billot, E. Pillet, E. Faouzi, et al., "Real-time queue-end detection on freeways with fcd: Practice: A practice-ready algorithm," in Transportation Research Board 93rd Annual Meeting, no. 14-2725, 2014.

[4] N. Consortium, "The harmonised research programme," www.nearctis.org, RoadMap D14, march 2012.

[5] I. G. Jin and G. Orosz, "Dynamics of connected vehicle systems with delayed acceleration feedback," Transportation Research Part C: Emerging Technologies, vol. 46, pp. 46-64, 2014.

[6] B. HomChaudhuri, R. Lin, and P. Pisu, "Hierarchical control strategies for energy management of connected hybrid electric vehicles in urban roads," Transportation Research Part C: Emerging Technologies, vol. 62, pp. 70-86, 2016.

[7] J. Ploeg, E. Semsar-Kazerooni, G. Lijster, N. van de Wouw, and H. Nijmeijer, "Graceful degradation of cooperative adaptive cruise control," Intelligent Transportation Systems, IEEE Transactions on, vol. 16, no. 1, pp. 488-497, 2015.

[8] O. A. Osman and S. Ishak, "A network level connectivity robustness measure for connected vehicle environments," Transportation Research Part C: Emerging Technologies, vol. 53, pp. 48-58, 2015.

[9] D. Ngoduy and R. Wilson, "Multianticipative nonlocal macroscopic traffic model," Computer-Aided Civil and Infrastructure Engineering, vol. 29, no. 4, pp. 248-263, 2014.

[10] J. Monteil, R. Billot, J. Sau, F. Armetta, S. Hassas, and N.-E. El Faouzi, "Cooperative highway traffic : multi-agent modeling and robustness assessment to local perturbations," Transportation Research Record: Journal of the Transportation Research Board, vol. 2391, no. 1, pp. 1-10, 2013.

[11] J. Monteil, R. Billot, J. Sau, and N.-E. El Faouzi, "Linear and weakly nonlinear stability analyses of cooperative car-following models," IEEE Transactions on Intelligent Transportation Systems, vol. pp, no. 99, pp. 1-13, 2014.

[12] J. Sau, J. Monteil, R. Billot, and N.-E. E. Faouzi, "The root locus method: application to linear stability analysis and design of cooperative car-following models," Transportmetrica B: Transport Dynamics, no. ahead-of-print, pp. 1-23, 2014.

[13] M. Treiber, A. Hennecke, and D. Helbing, "Congested traffic states in empirical observations and microscopic simulations," Physical Review E, vol. 62, no. 2, pp. 1805-1824, 2000.

[14] A. Kesting, M. Treiber, and D. Helbing, "General lane-changing model mobil for car-following models," Transportation Research Record: Journal of the Transportation Research Board, vol. 1999 / 2007 Traffic Flow Theory 2007, pp. 86-94, 2007.

[15] I. Zohdy and H. Rakha, "Game theory algorithm for intersection-based cooperative adaptive cruise control (cacc) systems," in Intelligent Transportation Systems (ITSC), 2012 15th International IEEE Conference on, Sept 2012, pp. 1097-1102.

[16] M. Treiber and A. Kesting, Traffic flow dynamics: data, models and simulation. Springer, 2013.

[17] A. Kesting, M. Treiber, and D. Helbing, "General lane-changing model mobil for car-following models," Transportation Research Record: Journal of the Transportation Research Board, vol. 1999, no. 1, pp. 86-94, 2007.

[18] H. X. Ge, S. Q. Dai, and L. Y. Dong, "An extended car-following model based on intelligent transportation system application," Physica A: Statistical Mechanics and its Applications, vol. 365, no. 2, pp. 543-548, 2006.

[19] D. Helbing and S. Balietti, Social Self-Organization. Springer, 2012, ch. Agent-Based Modeling, pp. 25-70.

[20] H. Zheng, Y.-J. Son, Y.-C. Chiu, L. Head, Y. Feng, H. Xi, S. Kim, and M. Hickman, "A primer for agent-based simulation and modeling in transportation applications," US Federal Highway Administration, Tech. Rep., 2013.

[21] M. Schillo, P. Funk, and M. Rovatsos, "Using trust for detecting deceitful agents in artificial societies," Applied Artificial Intelligence, vol. 14, no. 8, pp. 825-848, 2000.

[22] Q.-A. N. Vu, R. Canal, B. Gaudou, S. Hassas, and F. Armetta, "Trustsets: using trust to detect deceitful agents in a distributed information collecting system," Journal of Ambient Intelligence and Humanized Computing, vol. 3, no. 4, pp. 251-263, 2012.

[23] C. Castelfranchi and R. Falcone, "Principles of trust for mas: cognitive anatomy, social importance, and quantification," in Proceedings of the International Conference on Multi Agent Systems, 1998, pp. 72-79.

[24] I. Pinyol and J. Sabater-Mir, "Computational trust and reputation models for open multi-agent systems: a review," Artificial Intelligence Review, vol. 40, no. 1, pp. 1-25, 2013. [Online]. Available: http://dx.doi.org/10.1007/s10462-011-9277-z

[25] J. Monteil, R. Billot, F. Armetta, S. Hassas, and N.-E. El Faouzi, "Cooperative highway traffic : multi-agent modeling and robustness assessment to local perturbations," in the 92nd Annual Meeting of the Transportation Research Board, reviewed by TRB's Traffic Flow Theory and Characteristics Committee (AHB45), 2013.

[26] D. Krajzewicz, J. Erdmann, M. Behrisch, and L. Bieker, "Recent development and applications of SUMO - Simulation of Urban MObility," International Journal On Advances in Systems and Measurements, vol. 5, no. 3\&4, pp. 128-138, December 2012.

[27] J. Barceló and J. Casas, "Dynamic network simulation with aimsun," in Simulation approaches in transportation analysis. Springer, 2005, pp. 57-98.

[28] M. Fellendorf, "Vissim: A microscopic simulation tool to evaluate actuated signal control including bus priority," in 64th Institute of Transportation Engineers Annual Meeting, 1994, pp. 1-9. 
[29] C. Backfrieder, C. Mecklenbrauker, and G. Ostermayer, "Traffsim - a traffic simulator for investigating benefits ensuing from intelligent traffic management," in Modelling Symposium (EMS), 2013 European, Nov 2013, pp. 451-456.

[30] M. Treiber and A. Kesting, "An open-source microscopic traffic simulator," IEEE Intelligent Transportation Systems Magazine, vol. 2, no. 3, pp. 6-13, 2010.

[31] F. J. Martinez, C. K. Toh, J.-C. Cano, C. T. Calafate, and P. Manzoni, "A survey and comparative study of simulators for vehicular ad hoc networks (vanets)," Wireless Communications and Mobile Computing, vol. 11, no. 7, pp. 813-828, 2011.

[32] M. Balmer, M. Rieser, K. Meister, D. Charypar, N. Lefebvre, K. Nagel, and K. Axhausen, "Matsim-t: Architecture and simulation times," Multi-agent systems for traffic and transportation engineering, pp. 57-78, 2009.

[33] S. Espié, J. M. Auberlet, et al., "Archisim: A behavioral multi-actors traffic simulation model for the study of a traffic system including its aspects," International Journal of ITS Research, no. n1, 2007.

[34] M. Treiber and A. Kesting, Traffic Flow Dynamic : Data, Models and Simulation. Springer, 2013.

[35] A. Kesting, M. Treiber, and D. Helbing, Multi-Agent Systems: Simulation and Applications, 2009, ch. Agents for Traffic Simulation, pp. $325-356$.

[36] C. Sommer, R. German, and F. Dressler, "Bidirectionally Coupled Network and Road Traffic Simulation for Improved IVC Analysis," IEEE Transactions on Mobile Computing, vol. 10, no. 1, pp. 3-15, January 2011.

[37] M. Schönhof, M. Treiber, A. Kesting, and D. Helbing, "Autonomous detection and anticipation of jam fronts from messages propagated by intervehicle communication," Transportation Research Record: Journal of the Transportation Research Board, vol. 1999, no. 1, pp. 3-12, 2007.

[38] C. Thiemann, M. Treiber, and A. Kesting, "Longitudinal hopping in intervehicle communication: Theory and simulations on modeled and empirical trajectory data," Phys. Rev. E, vol. 78, p. 036102, Sep 2008. [Online]. Available: http://link.aps.org/doi/10.1103/PhysRevE.78.036102

[39] A. Kesting, M. Treiber, and D. Helbing, "Connectivity statistics of store-and-forward intervehicle communication," Intelligent Transportation Systems, IEEE Transactions on, vol. 11, no. 1, pp. 172-181, 2010.

[40] W. Van Willigen, E. Haasdijk, and L. Kester, "A multi-objective approach to evolving platooning strategies in intelligent transportation systems," in Proceeding of the fifteenth annual conference on Genetic and evolutionary computation conference. ACM, 2013, pp. $1397-1404$.

[41] X. Yan, F. Gu, X. Hu, and C. Engstrom, "Dynamic data driven event reconstruction for traffic simulation using sequential monte carlo methods," in Simulation Conference (WSC), 2013 Winter, Dec 2013, pp. 2042-2053.

[42] M. Guériau, R. Billot, N.-E. El Faouzi, H. Salima, and A. Frédéric, "Multi-agent dynamic coupling for cooperative vehicles modeling," in The Twenty-Ninth Conference on Artificial Intelligence AAAI'2015 - (DEMO Track), Austin, Texas, Jan 2015. [Online]. Available: http://youtu.be/uv60iu5hfMw

[43] J. Ferber, Multi-agent systems: an introduction to distributed artificial intelligence. Addison-Wesley Reading, 1999 , vol. 1.

[44] J. Monteil, R. Billot, J. Sau, C. Buisson, and N.-E. El Faouzi, "Calibration, estimation, and sampling issues of car-following parameters," Transportation Research Record: Journal of the Transportation Research Board, vol. 2422, no. 1, pp. 131-140, 2014.

[45] J. Choi, S. Oh, and R. Horowitz, "Distributed learning and cooperative control for multi-agent systems," Automatica, vol. 45, no. 12, pp. 2802-2814, Dec. 2009. [Online]. Available: http://dx.doi.org/10.1016/j.automatica.2009.09.025

[46] B. Van Arem, C. J. van Driel, and R. Visser, "The impact of cooperative adaptive cruise control on traffic-flow characteristics," Intelligent Transportation Systems, IEEE Transactions on, vol. 7, no. 4, pp. 429-436, 2006.

[47] M. Wang, W. Daamen, S. P. Hoogendoorn, and B. van Arem, "Rolling horizon control framework for driver assistance systems. part ii: Cooperative sensing and cooperative control," Transportation Research Part C: Emerging Technologies, vol. 40, pp. 290-311, 2014.

[48] T. D. Huynh, N. R. Jennings, and N. R. Shadbolt, "An integrated trust and reputation model for open multi-agent systems," Autonomous Agents and Multi-Agent Systems, vol. 13, no. 2, pp. 119-154, 2006. 